\title{
Interval Type-2 Fuzzy Sets are generalization of Interval-Valued Fuzzy Sets: Towards a Wider view on their relationship
}

\author{
Humberto Bustince, Javier Fernandez, Hani Hagras, Francisco Herrera, Miguel Pagola and Edurne Barrenechea
}

\begin{abstract}
In this paper, we will present a wider view on the relationship between interval-valued fuzzy sets and interval type2 fuzzy sets where we will show that interval-valued fuzzy sets are a particular case of the interval type-2 fuzzy sets. For this reason, both concepts should be treated in a different way. In addition, the view presented in this paper will allow a more general perspective of interval type-2 fuzzy sets which will allow representing concepts which could not be presented by intervalvalued fuzzy sets.
\end{abstract}

Index Terms-Interval-valued fuzzy sets; Interval type-2 fuzzy sets; Type-2 fuzzy sets.

\section{INTRODUCTION}

Since Zadeh introduced fuzzy sets in 1965 ([35]), researchers have realized that a key problem for using fuzzy sets is the construction of the membership grades. The difficulty arises from the uncertainty associated with allocating an exact numerical membership value for each element within the considered fuzzy set. For this reason, in 1971 Zadeh ([36]) suggested the notion of a type-2 fuzzy set (which was later formally introduced in [37]), where the membership degree of each element is given by another fuzzy set defined over the referential $[0,1]$.

In 1975, Sambuc ([29]) presented interval-valued fuzzy sets which can be used when there is a problem in determining the exact membership values of the given elements. In intervalvalued fuzzy sets, we may use intervals as membership values in such a way that the exact numerical membership degree is a value inside the considered interval.

In 2000, Liang and Mendel in their paper Interval type-2 fuzzy logic systems: Theory and design, [21] introduced the definition of an interval type-2 fuzzy set as a particular case of their mathematical formulation of the concept of a type- 2 fuzzy set where the secondary membership degree is equal to one.

In this paper, we will show that the notion of interval type-2 fuzzy set encompasses type-1 fuzzy sets, interval-valued fuzzy sets and other types of sets. Thus, the view presented in this

\footnotetext{
H. Bustince, J. Fernandez, M. Pagola and E. Barrenechea are with the Department of Automatic and Computation, Public University of Navarra, Pamplona, Spain, e-mail \{bustince,fcojavier.fernandez,miguel.pagola,edurne.barrenechea\}@ unavarra.es H. Hagras is with the Computational Intelligence Centre, School of Computer Science and Electronic Engineering, University of Essex, Colchester, UK, e-mail: hani@essex.ac.uk

F. Herrera is with the Department of Computation and Artificial Intelligence, CITIC-UGR, Universidad de Granada, Spain, e-mail: herrera@decsai.ugr.es
}

paper will allow a more general perspective of interval type-2 fuzzy sets which will allow representing concepts which could not be presented by interval-valued fuzzy sets.

This paper is organized as follows. In Section II, we recall the notions of type-1 fuzzy sets, interval-valued fuzzy sets, type-2 fuzzy sets and interval type-2 fuzzy sets. In Section III, we show how type-1 fuzzy sets and interval-valued fuzzy sets are particular cases of interval type- 2 fuzzy sets and we also present some examples of interval type-2 fuzzy sets which are neither type-1 fuzzy sets nor interval-valued fuzzy sets. Section IV presents the conclusions.

\section{TYPE-1 FUZZY SETS, INTERVAL-VALUED FUZZY SETS AND INTERVAL TYPE-2 FUZZY SETS}

In classical logic propositions an input can only be either true or false. Aristotle formulated the basic principles of this logic which encompass the non-contradiction principle (a statement can not be true and false at the same time) and the excluded middle principle (every statement is either true or false).

It is easy to note that there are many situations for which more than two truth values are needed. This fact led Peirce to say that Aristotle's formulation is the simplest hypothesis we can work with ([28]). In fact, as human knowledge representation is based upon concepts ([9]), and these concepts are not crisp in nature, hence, we should not expect that human beings use binary logic so often in their daily life. For instance, everyday perspectives such as taste, meaning of adjectives, etc. can only be studied precisely if we consider more complex gradings rather than the binary true or false grading.

These considerations have led to the proposal of different logical formulations which allowed for more than two truth values, like Brouwer's intuitionistic logic ([5]) (partially caught by the so called intuitionistic propositional calculus modeled by Heyting algebras) and multivalued logics presented by Lukasiewicz ([22]), or Zadeh's fuzzy logic (which replaces the set $\{0,1\}$ by the set $[0,1],[35])$.

\section{A. Type-1 Fuzzy sets}

Definition 1: A type-1 fuzzy set $A$ over a referential set $X$ can be written as follows:

$$
A=\left\{\left(x, \mu_{A}(x)\right) \mid x \in X\right\}
$$

where $\mu_{A}: X \longrightarrow[0,1]$. 
In this definition $\mu_{A}(x)$ represents the degree of membership of the element $x \in X$ to the set $A$. In this work we are going to use the following notation: $A(x)=\mu_{A}(x)$ for all $x \in X$.

As mentioned in [19], traditionally, a type-1 fuzzy set is not visualized in three dimensions, however, it is implicit in the definition of a type-1 fuzzy set that all the secondary grades in the third dimension are equal to 1 . This indicates that each primary membership grade $\left(\mu_{A}(x)\right)$ is fully certain (i.e. in a type-1 fuzzy set, for each $x$ value there is no uncertainty associated with the primary membership value) and therefore modelled as a crisp singleton. Thus, as all the secondary grades in the third dimension are equal 1, the third dimension is not visualized as it is redundant and does not present any information.

It is worth to mention that in 1967 ([12]) Goguen introduced the notion of $L$-fuzzy set, which extended the definition of fuzzy set by considering for truth values a lattice different from the unit interval $[0,1]$.

\section{B. Type-2 fuzzy sets}

In 1971, Zadeh suggested in his paper Quantitative fuzzy semantics ([36]) that the construction of the fuzzy set (which includes the allocation of the membership degree of each element to the fuzzy set) is the biggest problem for using fuzzy sets theory in real world applications. This fact led him to introduce the concept of a type-2 fuzzy set in the following way: A type-2 fuzzy set is a L-fuzzy set over a referential set $X$ for which the membership degrees of the elements are given by fuzzy sets defined over the referential set $[0,1]$ (see Fig. 1) (which is a lattice with respect to Zadeh's union and intersection operators, [36]).

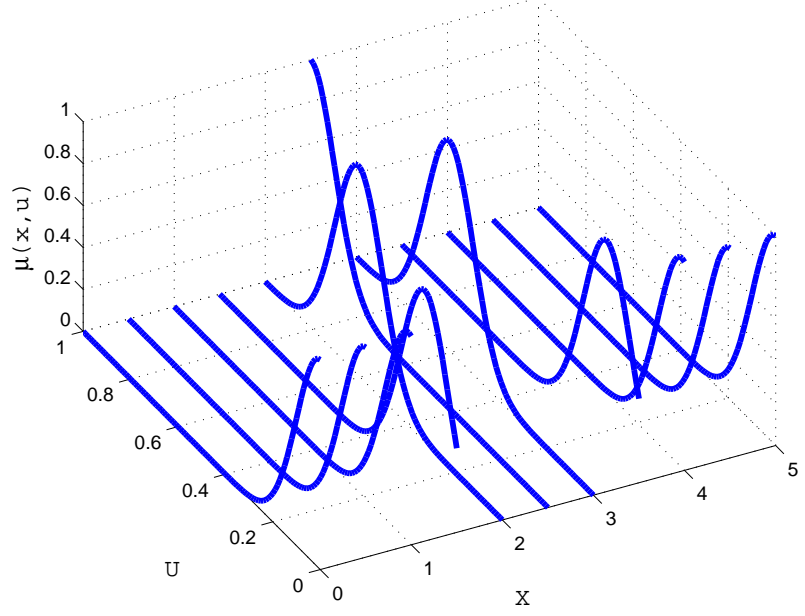

Fig. 1. Example of a type-2 fuzzy set

\section{Interval-valued fuzzy sets}

In 1975 Sambuc ([29]) presented in his doctoral thesis the concept of an interval-valued fuzzy set named a $\Phi$ fuzzy set. In the same year, Jahn ([18]) wrote about the notion of interval-valued fuzzy set. One year later, GrattanGuinness ([17]) established a definition of an interval-valued membership function. In that decade interval-valued fuzzy sets appeared in the literature in various guises and it was not until the 1980s, with the work of Gorzalczany and Turksen ([11], [13]-[16], [7], [30]-[32],[33]), that the importance of these sets, as well as their name, was definitely established.

Let us denote by $L([0,1])$ the set of all closed subintervals in $[0,1]$, that is,

$$
L([0,1])=\left\{\mathbf{x}=[\underline{x}, \bar{x}] \mid(\underline{x}, \bar{x}) \in[0,1]^{2} \text { and } \underline{x} \leq \bar{x}\right\} .
$$

Definition 2: An interval-valued fuzzy set $A$ on the universe $X \neq \emptyset$ is a mapping

$$
A: U \rightarrow L([0,1]),
$$

such that the membership degree of $x \in X$ is given by $A(x)=$ $[A(x), \bar{A}(x)] \in L([0,1])$, where $A: X \rightarrow[0,1]$ and $\bar{A}: X \rightarrow$ $[0,1]$ are mappings defining the lower and the upper bound of the membership interval $A(x)$, respectively (see Fig. 2).

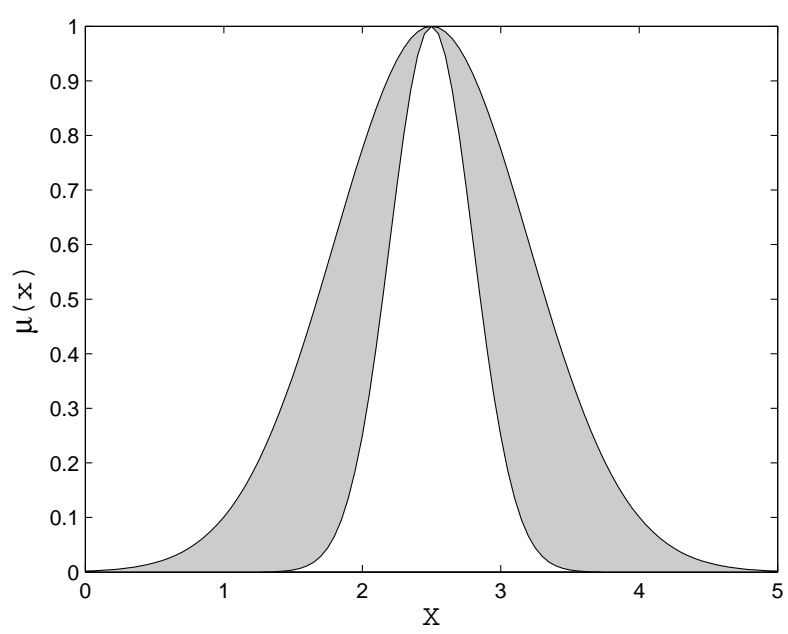

Fig. 2. Example of Interval-valued fuzzy set

Similar to the case of type-1 fuzzy sets, traditionally, an interval-valued fuzzy set is not visualized in three dimensions, however, it is implicit in the definition of an interval-valued fuzzy set that all the secondary grades in the third dimension are equal to 1 (i.e. each point in the interval has a secondary membership equal to 1 ). Thus, as all the secondary grades in the third dimension are equal 1, the third dimension is not visualized as it is redundant and does not present any information. Several different applications of these sets may be found in [10].

\section{Interval type-2 fuzzy sets}

Based on Zadeh's ideas, in 1976 Mizumoto and Tanaka ([25]) presented the mathematical definition of a type-2 fuzzy set. Since then, several authors have studied these sets using different notations. In particular, in 2006 Mendel, John and Liu defined these sets as follows ([24]): 
Definition 3: A type-2 fuzzy set, denoted $A$, on the universe $X \neq \emptyset$ is given by

$A=\left\{\left((x, u), \mu_{A}(x, u)\right) \mid\right.$ for all $x \in X$, for all $\left.u \in J_{x} \subseteq[0,1]\right\}$ in which $0 \leq \mu_{A}(x, u) \leq 1$.

Remark: This definition, including the notation, is directly extracted from [24].

From this definition, in 1999 (see [20]), Karnik, Mendel and Liang introduced the notion of interval type-2 fuzzy sets. In particular, Mendel, John and Liu defined interval type-2 fuzzy sets in 2006 (see [24], [23]) as follows:

Definition 4: When all $\mu_{A}(x, u)=1$ then $A$ is an interval type-2 fuzzy set.

For the operations of interval type- 2 fuzzy sets, meet and join operations should be used instead of union and intersection. Without meet and join operations, the fuzzifier in an interval type-2 fuzzy logic system would not work.

\section{Some SPECIAL CASES OF INTERVAL TYPE-2 FUZZY}

SETS

In this section we present some particular cases of Definition 4. For simplicity, we are going to work with a finite referential set $X$ of cardinal $m$. However, we could also deal with a nonfinite referential set.

Since $J_{x}$ is a subset of $[0,1]$; (that is $J_{x} \subseteq[0,1]$ ), in this work we are going to consider the following four special cases:

CASE (A) when $J_{x}=\left\{u_{x}\right\} \subseteq[0,1]$. It is easy to see that in this setting we recover type-1 fuzzy sets by taking:

$$
A(x)=u_{x} \in[0,1]
$$

Example 1: Let $X=\left\{x_{1}, x_{2}, x_{3}, x_{4}, x_{5}\right\}$ be the referential set. Consider the set:

$$
\begin{aligned}
& A=\left\{\left(x_{1}, \mu_{A}\left(x_{1}, u\right) \text { with } u \in J_{x_{1}}\right.\right. \\
& \left.\quad=\left\{u_{x_{1}}\right\}=\{0\} \subseteq[0,1]\right), \\
& \quad\left(x_{2}, \mu_{A}\left(x_{2}, u\right) \text { with } u \in J_{x_{2}}=\left\{u_{x_{2}}\right\}\right. \\
& \quad=\{0.55\} \subseteq[0,1]), \\
& \quad\left(x_{3}, \mu_{A}\left(x_{3}, u\right) \text { with } u \in J_{x_{3}}=\left\{u_{x_{3}}\right\}\right. \\
& \quad=\{1\} \subseteq[0,1]), \\
& \quad\left(x_{4}, \mu_{A}\left(x_{4}, u\right) \text { with } u \in J_{x_{4}}=\left\{u_{x_{4}}\right\}\right. \\
& \quad=\{0.5\} \subseteq[0,1]), \\
& \quad\left(x_{5}, \mu_{A}\left(x_{5}, u\right) \text { with } u \in J_{x_{5}}=\left\{u_{x_{5}}\right\}\right. \\
& \quad=\{0.2\} \subseteq[0,1])\}
\end{aligned}
$$

By Definition 4 we have (see Fig. 3):

$$
\begin{aligned}
& \mu_{A}\left(x_{1}, u\right)= \begin{cases}1 & \text { if } u=0 \\
0 & \text { if } u \neq 0\end{cases} \\
& \mu_{A}\left(x_{2}, u\right)= \begin{cases}1 & \text { if } u=0.55 \\
0 & \text { if } u \neq 0.55\end{cases} \\
& \mu_{A}\left(x_{3}, u\right)= \begin{cases}1 & \text { if } u=1 \\
0 & \text { if } u \neq 1\end{cases} \\
& \mu_{A}\left(x_{4}, u\right)= \begin{cases}1 & \text { if } u=0.5 \\
0 & \text { if } u \neq 0.5\end{cases} \\
& \mu_{A}\left(x_{5}, u\right)= \begin{cases}1 & \text { if } u=0.2 \\
0 & \text { if } u \neq 0.2\end{cases}
\end{aligned}
$$

Then we have the fuzzy set:

$$
\begin{gathered}
A=\left\{\left(x_{1}, A\left(x_{1}\right)=0\right),\left(x_{2}, A\left(x_{2}\right)=0.51\right),\left(x_{3}, A\left(x_{3}\right)=1\right),\right. \\
\left.\left(x_{4}, A\left(x_{4}\right)=0.5\right),\left(x_{5}, A\left(x_{5}\right)=0.2\right)\right\}
\end{gathered}
$$

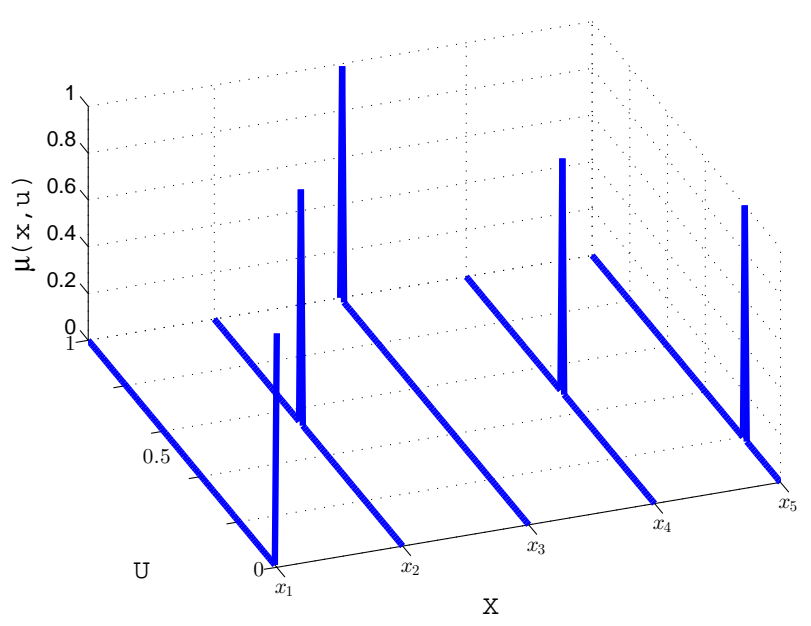

Fig. 3. Example of case A: an interval type-2 fuzzy set which is actually a type-1 fuzzy set

CASE (B) when $J_{x}=\left\{u_{x}^{1}, u_{x}^{2}, \cdots, u_{x}^{n}\right\} \subseteq[0,1]$ with $u_{x}^{i} \neq u_{x}^{j}$ if $i \neq j$.

Clearly, for different elements $x$ we may take different values of $n$. Notice that the sets defined in this way are a particular case of multisets where every membership is different from each other ([34], [4], [26]).

Example 2:

Let $X=\left\{x_{1}, x_{2}, x_{3}, x_{4}, x_{5}\right\}$ be the referential set. 
Consider the set:

$$
\begin{aligned}
& A=\left\{\left(x_{1}, \mu_{A}\left(x_{1}, u\right) \text { with } u \in J_{x_{1}}\right.\right. \\
& \left.\quad=\left\{u_{x_{1}}^{1}, u_{x_{1}}^{2}, u_{x_{1}}^{3}\right\}=\{0,0.05,0.1\} \subseteq[0,1]\right), \\
& \quad\left(x_{2}, \mu_{A}\left(x_{2}, u\right) \text { with } u \in J_{x_{2}}\right. \\
& \quad=\left\{u_{x_{2}}^{1}, u_{x_{2}}^{2}, u_{x_{2}}^{3}, u_{x_{2}}^{4}, u_{x_{2}}^{5}\right\}= \\
& \quad\{0.55,0.6,0.65,0.75\} \subseteq[0,1]), \\
& \left(x_{3}, \mu_{A}\left(x_{3}, u\right) \text { with } u \in J_{x_{3}}\right. \\
& \left.\quad=\left\{u_{x_{3}}^{1}, u_{x_{3}}^{2}\right\}=\{0.95,1\} \subseteq[0,1]\right), \\
& \left(x_{4}, \mu_{A}\left(x_{4}, u\right) \text { with } u \in J_{x_{4}}\right. \\
& \left.\quad=\left\{u_{x_{4}}^{1}, u_{x_{4}}^{2}, u_{x_{4}}^{3}\right\}=\{0.35,0.4,0.5\} \subseteq[0,1]\right), \\
& \left(x_{5}, \mu_{A}\left(x_{5}, u\right) \text { with } u \in J_{x_{5}}\right. \\
& \left.\left.\quad=\left\{u_{x_{5}}^{1}, u_{x_{5}}^{2}, u_{x_{5}}^{3}\right\}=\{0.2,0.25,0.3\} \subseteq[0,1]\right)\right\}
\end{aligned}
$$

By Definition 4 we have (see Fig. 4):

$$
\begin{aligned}
& \mu_{A}\left(x_{1}, u\right)= \begin{cases}1 & \text { if } u \in\{0,0.05,0.1\} \\
0 & \text { otherwise }\end{cases} \\
& \mu_{A}\left(x_{2}, u\right)= \begin{cases}1 & \text { if } u \in\{0.55,0.6,0.65,0.75\} \\
0 & \text { otherwise }\end{cases} \\
& \mu_{A}\left(x_{3}, u\right)= \begin{cases}1 & \text { if } u \in\{0.95,1\} \\
0 & \text { otherwise }\end{cases} \\
& \mu_{A}\left(x_{4}, u\right)= \begin{cases}1 & \text { if } u \in\{0.35,0.4,0.5\} \\
0 & \text { otherwise }\end{cases} \\
& \mu_{A}\left(x_{5}, u\right)= \begin{cases}1 & \text { if } u \in\{0.2,0.25,0.3\} \\
0 & \text { otherwise }\end{cases}
\end{aligned}
$$

Then, we have the multiset:

$$
\begin{aligned}
A=\{ & \left(x_{1}, 0,0.5,0.1\right),\left(x_{2}, 0.56,0.6,0.69,0.75\right) \\
& \left.\left(x_{3}, 0.95,1\right),\left(x_{4}, 0.35,0.4,0.5\right),\left(x_{5}, 0.2,0.25,0.3\right)\right\}
\end{aligned}
$$

Note that this example may correspond, for instance, to a setting in which several anonymous users of a web recommendation system provide their valuations for a given object. In this situation (and due to anonymity, on the one hand, and the fact that not every user of the system may vote for every object), sets with more than one membership value arise.

CASE (C) when $J_{x}=\left[A_{x}, \overline{A_{x}}\right] \subseteq[0,1]$. In this setting, we recover interval-valued fuzzy sets. Taking into account the mathematical equivalence between intervalvalued fuzzy sets and Atanassov's intuitionistic fuzzy sets ([1], [2]), with $J_{x}=\left[A_{x}, \overline{A_{x}}\right] \subseteq[0,1]$ we also recover Atanassov intuitionistic fuzzy sets. Note that this mathematical equivalence is defined as follows: given an interval $\left[A_{x}, \overline{A_{x}}\right]$, we take $\mu_{A}(x)=A_{x}$ and $\nu_{A}(x)=1-\overline{A_{x}}$. In this way, $\mu_{A}(x)+\nu_{A}(x) \overline{\overline{1}}$.

Example 3: Let $X=\left\{x_{1}, x_{2}, x_{3}, x_{4}, x_{5}\right\}$ be the refer-

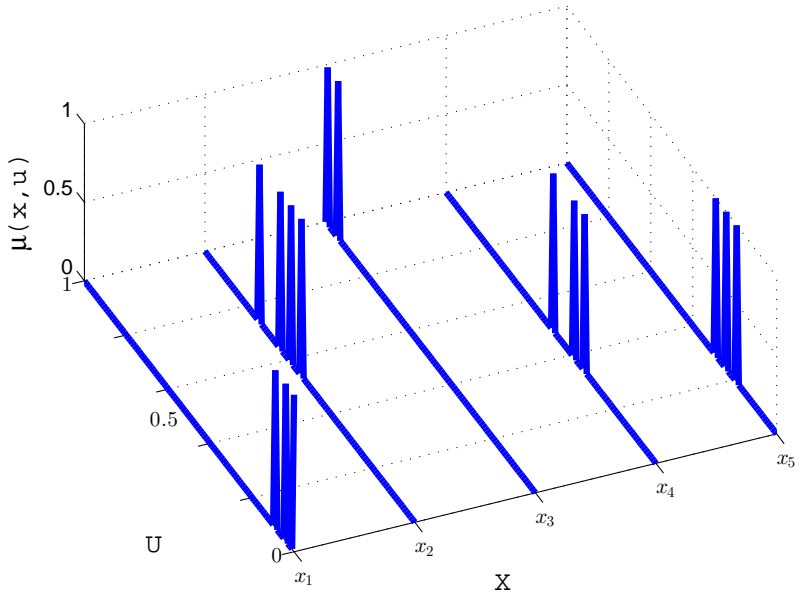

Fig. 4. Example of a multiset from an interval type-2 fuzzy set

ential set. Consider the set:

$$
\begin{aligned}
& A=\left\{\left(x_{1}, \mu_{A}\left(x_{1}, u\right) \text { with } u \in J_{x_{1}}\right.\right. \\
& \left.\quad=\left[\underline{A_{x_{1}}}, \overline{A_{x_{1}}}\right]=[0,0.2] \subseteq[0,1]\right), \\
& \quad\left(x_{2}, \mu_{A}\left(x_{2}, u\right) \text { with } u \in J_{x_{2}}\right. \\
& \left.\quad=\left[\underline{A_{x_{2}}}, \overline{A_{x_{2}}}\right]=[0.2,0.6] \subseteq[0,1]\right), \\
& \quad\left(x_{3}, \mu_{A}\left(x_{3}, u\right) \text { with } u \in J_{x_{3}}\right. \\
& \left.\quad=\left[\underline{A_{x_{3}}}, \overline{A_{x_{3}}}\right]=[0.7,1] \subseteq[0,1]\right), \\
& \quad\left(x_{4}, \overline{\mu_{A}}\left(x_{4}, u\right) \text { with } u \in J_{x_{4}}\right. \\
& \left.\quad=\left[\underline{A_{x_{4}}}, \overline{A_{x_{4}}}\right]=[0.5,0.8] \subseteq[0,1]\right), \\
& \quad\left(x_{5}, \mu_{A}\left(x_{5}, u\right) \text { with } u \in J_{x_{5}}\right. \\
& \left.\left.\quad=\left[\underline{A_{x_{5}}}, \overline{A_{x_{5}}}\right]=[0.1,0.4] \subseteq[0,1]\right)\right\}
\end{aligned}
$$

By Definition 4 we have (see Fig. 5):

$$
\begin{aligned}
& \mu_{A}\left(x_{1}, u\right)= \begin{cases}1 & \text { if } u \in[0,0.2] \\
0 & \text { otherwise }\end{cases} \\
& \mu_{A}\left(x_{2}, u\right)= \begin{cases}1 & \text { if } u \in[0.2,0.6] \\
0 & \text { otherwise }\end{cases} \\
& \mu_{A}\left(x_{3}, u\right)= \begin{cases}1 & \text { if } u \in[0.7,1] \\
0 & \text { otherwise }\end{cases} \\
& \mu_{A}\left(x_{4}, u\right)= \begin{cases}1 & \text { if } u \in[0.5,0.8 \\
0 & \text { otherwise }\end{cases} \\
& \mu_{A}\left(x_{5}, u\right)= \begin{cases}1 & \text { if } u \in[0.1,0.4] \\
0 & \text { otherwise }\end{cases}
\end{aligned}
$$

then we have the interval-valued fuzzy set:

$$
\begin{aligned}
& A=\left\{\left(x_{1},[0,0.2]\right),\left(x_{2},[0.2,0.6]\right),\left(x_{3},[0.7,1]\right),\right. \\
&\left.\left(x_{4},[0.5,0.8]\right),\left(x_{5},[0.1,0.4]\right)\right\}
\end{aligned}
$$

Note that interval valued fuzzy sets naturally arise in those situation where an expert is not able to provide a precise membership value for each of the elements of a fuzzy sets ([3]). 


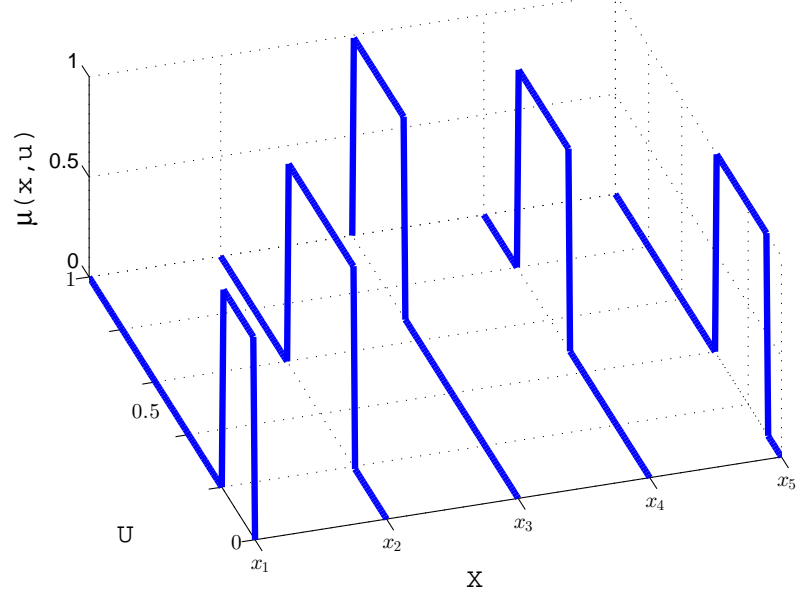

Fig. 5. Example of an interval-valued fuzzy set as an interval type-2 fuzzy set

$$
\begin{aligned}
& \mu_{A}\left(x_{1}, u\right)= \begin{cases}1 & \text { if } u \in[0,0.2] \cup[0.25,0.3] \\
0 & \text { otherwise }\end{cases} \\
& \mu_{A}\left(x_{2}, u\right)= \begin{cases}1 & \text { if } u \in[0.4,0.5] \cup[0.65,0.70] \cup \\
0 & 0.75,0.85]\end{cases} \\
& \mu_{A}\left(x_{3}, u\right)= \begin{cases}1 & \text { otherwise } u \in[0.7,1] \\
0 & \text { otherwise }\end{cases} \\
& \mu_{A}\left(x_{4}, u\right)= \begin{cases}1 & \text { if } u \in[0.5,0.6] \cup[0.7,0.8] \\
0 & \text { otherwise }\end{cases} \\
& \mu_{A}\left(x_{5}, u\right)= \begin{cases}1 & \text { if } u \in[0.1,0.2] \cup[0.25,0.5] \\
0 & \text { otherwise }\end{cases}
\end{aligned}
$$

Then we have a particular case of multi interval-valued fuzzy set:

$$
\begin{aligned}
A=\{ & \left(x_{1},[0,0.2],[0.25,0.3]\right),\left(x_{2},[0.4,0.5],[0.65,0.70],\right. \\
& {[0.75,0.85]),\left(x_{3},[0.7,1]\right),\left(x_{4},[0.5,0.6],[0.7,0.8]\right), } \\
& \left.\left(x_{5},[0.1,0.2],[0.25,0.5]\right)\right\}
\end{aligned}
$$

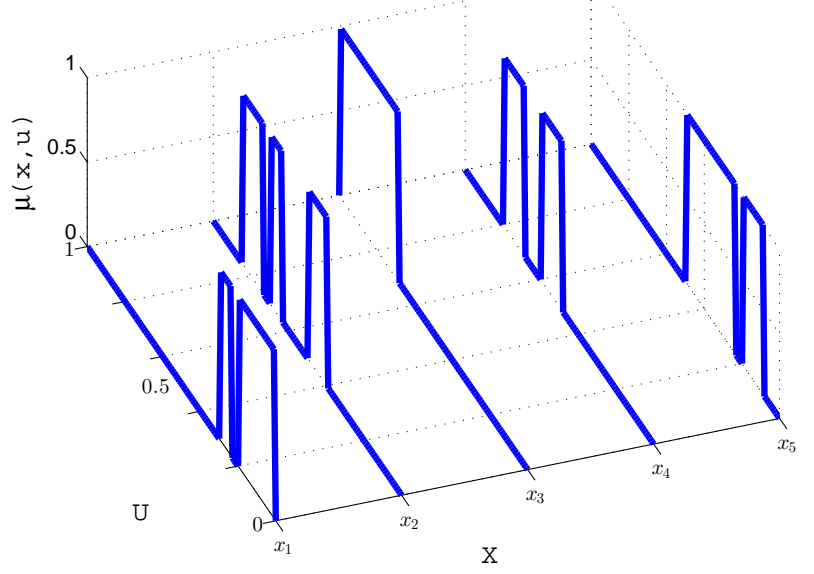

Fig. 6. Example of a multi interval-valued fuzzy set as an interval type-2 fuzzy set

Note that this case can be seen, from the application point of view as a combination of all the previously mentioned examples.

We can also consider more other particular cases for $J_{x}$. However, we consider that with (A)-(D) the goal of this work has been reached. However, we consider that it is important to mention the following setting: $J_{x}=$ $\left[u_{x}^{1}, u_{x}^{1}\right] \cup\left[A_{x}^{2}, \overline{A_{x}^{2}}\right] \cup\left[u_{x}^{3}, u_{x}^{3}\right] \cup \cdots \subseteq[0,1]$, which is a particular case of (D) and which we analyze as Case (E) described below.

CASE (E) when $J_{x}=\left[\underline{A_{x}^{1}}, \overline{A_{x}^{1}}\right] \cup\left[\underline{\underline{A_{x}^{2}}}, \overline{A_{x}^{2}}\right] \cup \cdots \cup$ $\left[A_{x}^{n}, \overline{A_{x}^{n}}\right] \subseteq[0,1]$ with $\left[A_{x}^{i}, \overline{\overline{A_{x}^{i}}}\right] \cap\left[A_{x}^{j}, \overline{\overline{A_{x}^{j}}}\right]=\emptyset$ if $i \neq j$ where some of the intervals degenerate into a single point; that is, pairwise disjoint intervals and points.

By Definition 4 we have (see Fig. 6): 
Example 5: Let $X=\left\{x_{1}, x_{2}, x_{3}, x_{4}, x_{5}\right\}$ be the referential set. Consider the set:

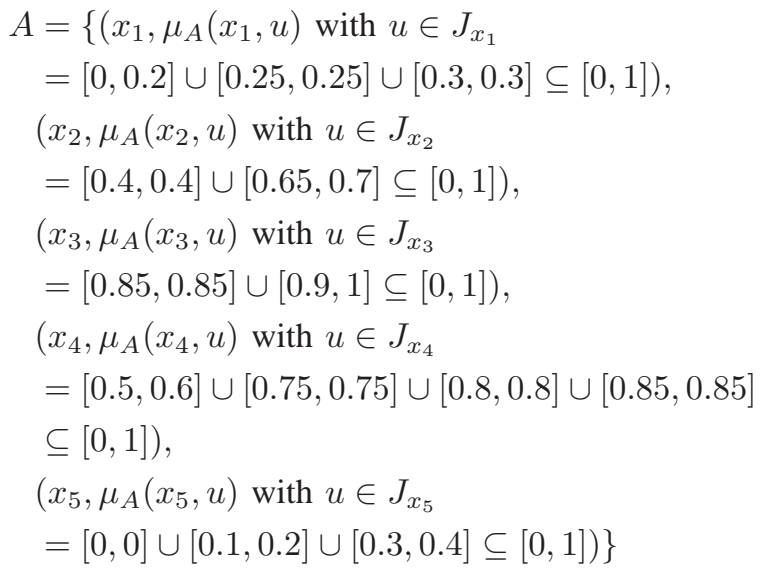

By Definition 4 we have (see Fig. 7):

$$
\begin{aligned}
& \mu_{A}\left(x_{1}, u\right)= \begin{cases}1 & \text { if } u \in[0,0.2] \cup[0.25,0.25] \\
0 & \cup[0.3,0.3]\end{cases} \\
& \mu_{A}\left(x_{2}, u\right)= \begin{cases}1 & \text { if } u \in[0.4,0.4] \cup[0.65,0.7] \\
0 & \text { otherwise }\end{cases} \\
& \mu_{A}\left(x_{3}, u\right)= \begin{cases}1 & \text { if } u \in[0.85,0.85] \cup[0.9,1] \\
0 & \text { otherwise }\end{cases} \\
& \mu_{A}\left(x_{4}, u\right)= \begin{cases}1 & \text { if } u \in[0.5,0.6] \cup[0.75,0.75] \\
0 & \cup[0.8,0.8] \cup[0.85,0.85]\end{cases} \\
& \mu_{A}\left(x_{5}, u\right)= \begin{cases}1 & \text { if } u \in[0,0] \cup[0.1,0.2] \\
0 & \cup[0.3,0.4]\end{cases}
\end{aligned}
$$

Then we have:

$$
\begin{aligned}
A=\{ & \left(x_{1},[0,0.2],[0.25,0.25],[0.3,03]\right), \\
& \left(x_{2},[0.4,0.4],[0.65,0.7]\right),\left(x_{3},[0.85,0.85],[0.9,1]\right), \\
& \left(x_{4},[0.5,0.6],[0.75,0.75],[0.8,0.8],[0.85,0.85]\right), \\
& \left.\left(x_{5},[0,0],[0.1,0.2],[0.3,0.4]\right)\right\}
\end{aligned}
$$

\section{CONClusions AND Future Researchs}

In this paper we have shown that interval type-2 fuzzy sets as defined in [21] are more general than interval-valued fuzzy sets. Hence, the definition introduced by Liang and Mendel generalizes the one given by Sambuc in 1975. Moreover we have presented four particular cases of interval type- 2 fuzzy sets:

- Type-1 fuzzy sets (Case (A)),

- a particular case of multi fuzzy set (Case (B)),

- interval-valued fuzzy sets (Case (C)),

- a particular case of multi interval-valued fuzzy set(Cases (D) and (E)).

Clearly, there are more special cases, depending on the subsets $J_{x}$ that we take.

It is important to note that in most of the papers where authors say they are using interval type-2 fuzzy sets, they are in

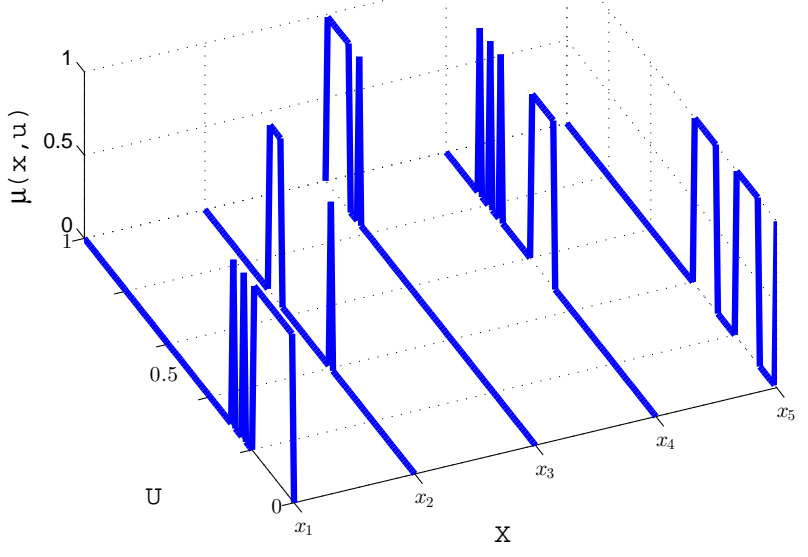

Fig. 7. Example of a multi interval-valued fuzzy set as an interval type-2 fuzzy set

fact taking for each $x \in X$ the subset $J_{x}=\left[A_{x}, \overline{A_{x}}\right] \subseteq[0,1]$ (Case (C)), so they are actually working with interval-valued fuzzy sets. Hence, we have presented a wider view on the relationship between interval-valued fuzzy Sets and interval type-2 fuzzy sets where we showed that interval-valued fuzzy sets are a particular case of the interval type- 2 fuzzy sets. In addition, the view presented in this paper will allow a more general perspective of interval type- 2 fuzzy sets. This view will enable us to represent concepts which could not be presented by interval-valued fuzzy sets.

Figure 8 shows graphically the relation between the different extensions considered in the present work and in Table I we summarize the different cases.

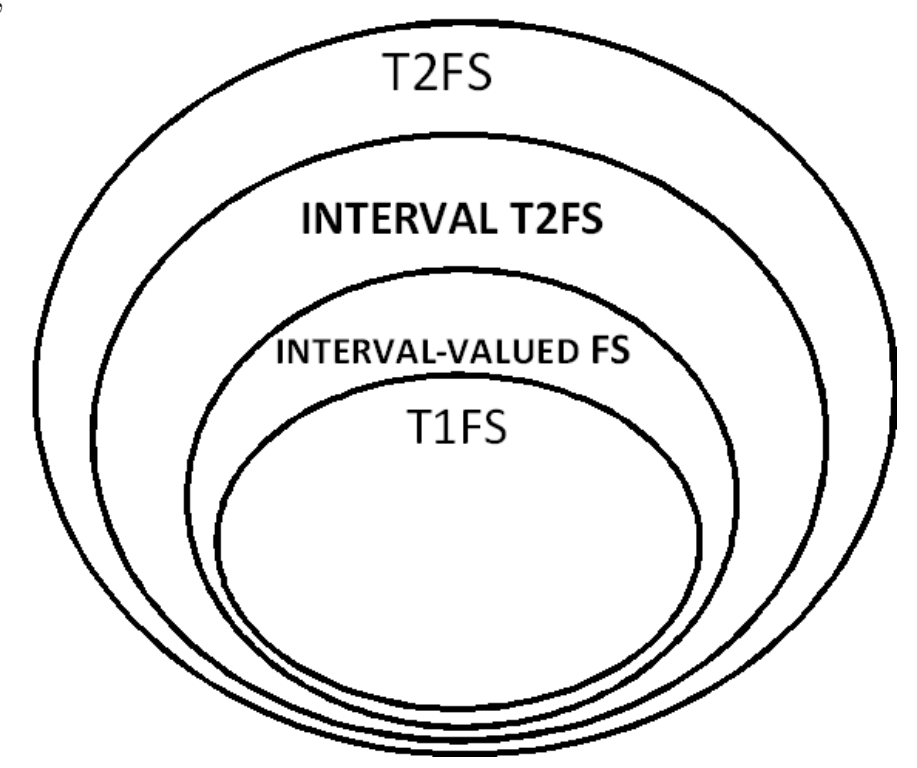

Fig. 8. Multi-Valued Logic Descriptive Power Hierarchy 
TABLE I

SUMMARY OF THE DIFFERENT CASES

\begin{tabular}{|c|c|}
\hline Type of fuzzy set & $J_{x}$ is a subset of $[0,1]$, that is, $J_{x} \subseteq[0,1]$ \\
\hline Type-1 fuzzy set & $J_{x}=\left\{u_{x}\right\} \subseteq[0,1]$ \\
\hline A particular case of multiset & $J_{x}=\left\{u_{x}^{1}, u_{x}^{2}, \cdots, u_{x}^{n}\right\} \subseteq[0,1]$ with $u_{x}^{i} \neq u_{x}^{j}$ if $i \neq j$ \\
\hline Interval-valued fuzzy set & $J_{x}=\left[\underline{A_{x}}, \overline{A_{x}}\right] \subseteq[0,1]$ \\
\hline $\begin{array}{l}\text { A particular case of multi } \\
\text { interval-valued fuzzy set }\end{array}$ & $\begin{array}{l}J_{x}=\left[\underline{A_{x}^{1}}, \overline{A_{x}^{1}}\right] \cup\left[\underline{A_{x}^{2}}, \overline{A_{x}^{2}}\right] \cup \cdots \cup\left[\underline{A_{x}^{n}}, \overline{A_{x}^{n}}\right] \subseteq[0,1] \\
\text { with }\left[\underline{\underline{A_{x}^{i}}}, \overline{A_{x}^{i}}\right] \cap\left[\underline{\underline{A_{x}^{j}}}, \overline{A_{x}^{j}}\right]=\emptyset \text { if } i \neq j\end{array}$ \\
\hline $\begin{array}{l}\text { A combination of numbers and } \\
\text { intervals }\end{array}$ & $\begin{array}{l}J_{x}=\left[u_{x}^{1}, u_{x}^{1}\right] \cup\left[\underline{A_{x}^{2}}, \overline{A_{x}^{2}}\right] \cup\left[u_{x}^{3}, u_{x}^{3}\right] \cup \cdots \subseteq[0,1] \text { with }\left[\underline{A_{x}^{i}}, \overline{A_{x}^{i}}\right] \cap\left[\underline{A_{x}^{j}}, \overline{A_{x}^{j}}\right]= \\
\emptyset \text { if } i \neq j\end{array}$ \\
\hline
\end{tabular}

In a future research paper we will study how the hierarchical relationships among the different multi-valued logics can affect the inference capabilities of each logic. In particular, we will analyze if the descriptive power is directly related to the inference power of the logics.

\section{Acknowledgments}

This work was supported in part by the Spanish Ministry of Science and Technology under Project TIN2011-28488 and Project TIN2013-40765-P.

\section{REFERENCES}

[1] K.T. Atanassov, "Intuitionistic fuzzy sets", Fuzzy Sets and Systems, vol. 20, pp. 87-96, 1986.

[2] K. T. Atanassov, G. Gargov, "Interval valued intuitionistic fuzzy sets", Fuzzy Sets and Systems, vol. 31(3), pp. 343-349, 1989.

[3] E. Barrenechea, H. Bustince, B. De Baets, C. Lopez-Molina,, "Construction of interval-valued fuzzy relations with application to the generation of fuzzy edge images", IEEE Transactions on Fuzzy Systems, vol. 19(5), pp. 819-830, 2011.

[4] B. Bedregal, G. Beliakov, H. Bustince, T. Calvo, R. Mesiar, D. Paternain, "A class of fuzzy multisets with a fixed number of memberships", Information Sciences, vol. 189, pp. 1-17, 2012.

[5] L. E. J. Brouwer, "Intuitionism and Formalism", Bulletin of the American Mathematical Society, vol. 20 (2), pp. 81-96, 1913.

[6] H. Bustince, 'Indicator of inclusion grade for interval-valued fuzzy sets. Application to approximate reasoning based on interval-valued fuzzy sets", International Journal of Approximate Reasoning, vol. 23(3), pp. 137-209, 2000.

[7] H. Bustince, J. Montero, M. Pagola, E. Barrenechea, D.Gómez, "A survey of interval-valued fuzzy sets", in: W. Pedrycz (Ed.), Handbook of Granular Computing, Wiley, New Jersey, 2008.

[8] H. Bustince, F. Herrera, J. Montero, Fuzzy Sets and Their Extensions: Representation Aggregation and Models, Springer, Berlin, 2007.

[9] J. T. Cacioppo, W. L. Gardner, C.G. Berntson, "Beyond bipolar conceptualizations and measures. The case of attitudes and evaluative space", Personality and Social Psychology Review, vol. 1, pp. 3-25, 1997.

[10] D. Dubois, H. Prade, R.R. Yager, Fuzzy information engineering. A guided tour of applications, John Wiley \& Sons, New York, 1997.

[11] A. Dziech, M. B. Gorzalczany, "Decision making in signal transmission problems with interval-valued fuzzy sets", Fuzzy Sets and Systems, vol. 23(2), pp.191-203, 1987.

[12] J. Goguen, "L-Fuzzy sets", Journal of Mathematical Analysis and Applications, vol. 18, pp. 145-174, 1967.

[13] M. B. Gorzalczany, "A method of inference in approximate reasoning based on interval-valued fuzzy sets"', Fuzzy Sets and Systems, vol. 21, pp. 1-17, 1987.

[14] M. B. Gorzalczany, "Interval-valued fuzzy controller based on verbal model of object", Fuzzy Sets and Systems, vol. 28(1), pp. 45-53, 1988.

[15] M. B. Gorzalczany, "Interval-valued fuzzy inference involving uncertain (inconsistent) conditional propositions", Fuzzy Sets and Systems, vol. 29(2), pp. 235-240, 1989.
[16] M. B. Gorzalczany, "An interval-valued fuzzy inference method. Some basic properties", Fuzzy Sets and Systems, vol. 31(2), pp. 243-251, 1989.

[17] I. Grattan-Guinness, "Fuzzy membership mapped onto interval and many-valued quantities", Zeitschrift für mathematische Logik und Grundladen der Mathematik, vol. 22, pp. 149-160, 1976.

[18] K. U. Jahn, "Intervall-wertige Mengen", Mathematische Nachrichten, vol. 68 , pp. 115-132, 1975.

[19] R. John, S. Coupland, Type-2 Fuzzy Logic: Challenges and Misconceptions, IEEE Computational Intelligence Magazine, pp. 48-52, August 2012.

[20] N. N. Karnik, J. M. Mendel, Q. Liang, "Type-2 fuzzy logic systems", IEEE Transactions on Fuzzy Systems, vol. 7(6), pp. 643-658, 1999.

[21] Q. Liang, J. Mendel, "Interval type-2 fuzzy logic systems: Theory and design", IEEE Transactions on Fuzzy Systems, vol. 8,pp. 535-550, 2000.

[22] J. Lukasiewicz, Elements of Mathematical Logic, Panstwowe Wydawnictwo Naukowe, Warsaw (Poland), 1963.

[23] J. Mendel, Uncertain Rule-Based Fuzzy Logic Systems: Introduction and New Directions, Upper Saddle River, NJ: Prentice-Hall, 2001.

[24] J. M. Mendel, R. I. John, F. Liu, "Interval type-2 fuzzy logic systems made simple", IEEE Transactions on Fuzzy Systems, vol. 14(6), pp. 808821, 2006.

[25] M. Mizumoto, K. Tanaka, K., "Some properties of fuzzy sets of type 2", Information and Control, vol. 31, pp. 312-340, 1976.

[26] S. Miyamoto, "Multisets and fuzzy multisets", in Z.-Q. Liu, S. Miyamoto (Eds.), Soft Computing and Human-Centered Machines, pp. 9-33, Springer, Berlin, 2000.

[27] M. Pagola, C. Lopez-Molina, J. Fernandez, E. Barrenechea, H. Bustince, "Interval type-2 fuzzy sets constructed from several membership functions. Application to the fuzzy thresholding algorithm", IEEE Transactions on Fuzzy Systems, vol. 21(2), pp. 230-244, 2013.

[28] C.S. Peirce, Collected Papers of Charles Sanders Peirce, Harvard University Press, 1958.

[29] R. Sambuc, "Function $\Phi$-Flous, Application a l'aide au Diagnostic en Pathologie Thyroidienne", These de Doctorat en Medicine, University of Marseille, 1975.

[30] I. B. Türksen, 'Interval valued fuzzy sets based on normal forms", Fuzzy Sets and Systems, vol. 20(2), pp. 191-210, 1986.

[31] I. B. Türksen, "Interval-valued fuzzy sets and compensatory AND", Fuzzy Sets and Systems, vol. 51, pp.295-307, 1992.

[32] I. B. Türksen, Z. Zhong, "An approximate analogical reasoning schema based on similarity measures and interval-valued fuzzy sets", Fuzzy Sets and Systems, vol. 34, pp. 323-346, 1990.

[33] I. B. Türksen, D.D. Yao, "Representation of connectives in fuzzy reasoning: The view through normal forms", IEEE Transactions on Systems, Man and Cybernetics, vol. 14, pp. 191-210, 1984.

[34] R.R. Yager, "On the theory of bags", International Journal of General Systems, vol. 13, pp. 23-37, 1986.

[35] L. A. Zadeh, "Fuzzy Sets", Information and Control, vol. 8, pp.338-353, 1965.

[36] L. A. Zadeh, "Quantitative fuzzy semantics", Information Sciences, vol. 3, pp. 159-176, 1971.

[37] L. A. Zadeh, "The concept of linguistic variable and its application to approximate reasoning-I", Information Sciences, vol. 8(3), pp. 199-249, 1975. 